\title{
Correlations in the Sequence of Residence Times
}

\author{
Benjamin Lindner \\ Max-Planck-Institut für Physik komplexer Systeme, Nöthnitzer Strasse 38, 01187 Dresden, Germany
}

Tilo Schwalger

Humboldt-Universität Berlin, Newtonstrasse 15, 12489 Berlin, Germany

(Received 17 January 2007; published 24 May 2007)

\begin{abstract}
Sequences of residence times (RTs) associated with the escape from metastable states are observed in many fields. Here we study analytically and numerically the correlations among RTs for a bistable stochastic system driven by dichotomous noise. Our theory predicts an oscillatory behavior of the correlations with respect to the lag between RTs. Correlations vanish at all lags if the switching rate matches the hopping rate of the unperturbed system. It is also shown that RT correlations may reveal features of the driving which are not present in the single-RT statistics.
\end{abstract}

PACS numbers: 05.40.-a, 05.10.Gg

The activated escape from a metastable state is an important problem encountered in various scientific fields such as chemical physics, solid state physics, and biological physics [1]. Starting with the pioneering work of Kramers [2], researchers have extensively studied the mean of the residence time (RT) in the metastable state and its inverse, the escape rate, in different situations, taking into account noise correlations [3], external periodic [4] or stochastic driving [5], etc. In some simple cases, higher order statistics of the single-RT time have also been analytically calculated (see, e.g., [6]).

Not much attention has been paid to the fact that RTs are most naturally observed in sequences: the subsequent epochs spent by a Brownian particle in the left and right wells of a quartic potential, the time spent in the neighboring minima of a periodic potential, or the periods spent near the resting state of an excitable system [7] -all of these residence times are commonly measured (in experiments or in a model simulation) as an ordered sequence $\left\{I_{k}\right\}$, with $k=0,1, \ldots$. These RTs will be only in the most simple cases mutually independent. In any case involving external driving, inertia, delayed feedback, etc., we expect that RT correlations will emerge. Except for certain neuron models where sequences of interspike intervals correspond to sequences of first-passage times [8-10], however, such correlations in the RT sequence have not been studied to the best of our knowledge.

In this Letter, we study RT correlations in a simple setup that is analytically tractable. For a bistable system driven by a thermal and by a dichotomous noise, the standard statistics of the RT (i.e., the RT density) will be contrasted with the surprising features of the RT correlation statistics. We show that, besides the expected behavior of correlations at the small switching rate of the dichotomous driving, we find nonintuitive effects such as the vanishing of linear correlations when the driving's switching rate matches the unperturbed escape rate of the system and an inversion of the RT correlation's sign for an even larger switching rate of the driving. We also demonstrate how input parameters can be inferred from the RT correlations if the driving is slow and weak.

We consider the dynamics of an overdamped bistable system driven by a dichotomous (telegraph) noise:

$$
\dot{x}=-U^{\prime}(x)+\eta(t)+\sqrt{2 D} \xi(t) .
$$

Here $U^{\prime}(x)$ is the spatial derivative of a quartic potential $U(x)=x^{4} / 4-x^{2} / 2$ with a potential barrier $\Delta U=1 / 4$ separating the two minima at $x= \pm 1 . \xi(t)$ is a white Gaussian noise, with $\left\langle\xi(t) \xi\left(t^{\prime}\right)\right\rangle=\delta\left(t-t^{\prime}\right)$. The dichotomous noise $\eta(t)$ switches between $\sigma$ and $-\sigma$ with rate $\lambda$ and possesses an exponential correlation function $\left\langle\eta(t) \eta\left(t^{\prime}\right)\right\rangle=\sigma^{2} \exp \left[-2 \lambda\left|t-t^{\prime}\right|\right]$ with a correlation time $1 /(2 \lambda)$. We consider the case of weak noise and weak driving where $\sigma \ll \Delta U$. In this case, the minima of the effective potential $U(x)-x \eta$ are approximately still at $x= \pm 1$, and the potential barrier will be different as seen from the two wells, namely, $\Delta U \pm \eta$. The model in Eq. (1) can serve as a case study of a nonlinear system driven by a correlated noise [3]; it has also been studied in the context of stochastic synchronization [11]; it may, furthermore, also be regarded as a model for two bistable systems being unidirectionally coupled (such systems have been studied in the context of noise-mediated patterns [12]). Possible experimental realizations include noisy electronic circuits [13], flux gate magnetometers [12], and bistable interference filters [14].

The dynamics equation (1) can be easily simulated, and a long sequence of residence times $\left\{I_{k}\right\}$ can be measured as indicated in Fig. 1. In a similar way, one could obtain a RT sequence from experimental time series: (i) Find the instants of switchings between metastable states (using, for instance, a threshold criterion); (ii) define the sequence of residence times as a sequence of differences between adjacent switching times. 


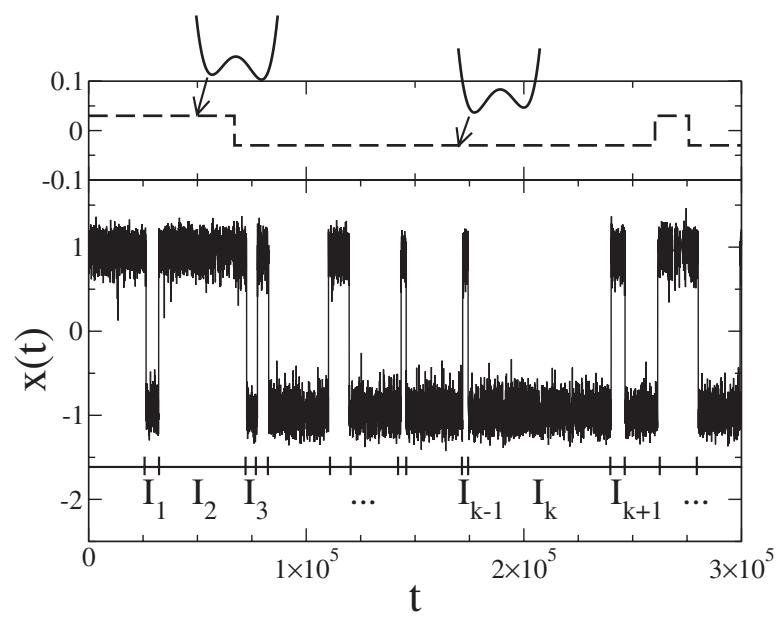

FIG. 1. Trajectory of the system Eq. (1) for a slow driving of the system $\left(\lambda=10^{-5}, D=\sigma=0.03\right)$ showing bistable switchings (middle trace). The dichotomous noise (upper trace) introduces an asymmetry in the effective potential (indicated in the upper part) and thus in the length of RT in the left and right wells. For piecewise constant parts of the dichotomous noise, one observes sequences of RTs (the intervals in the lower trace) alternating between short and long values.

Correlations of the $I_{k}$ are commonly characterized by the serial correlation coefficient (SCC)

$$
\rho_{n}=\frac{\left\langle I_{k+n} I_{k}\right\rangle-\left\langle I_{k+n}\right\rangle\left\langle I_{k}\right\rangle}{\left\langle I_{k}^{2}\right\rangle-\left\langle I_{k}\right\rangle^{2}}
$$

which is the covariance of the two RTs $I_{k}$ and $I_{k+n}$ normalized by the variance of the single RT. The average $\langle\cdot\rangle$ is performed over the sequence $\left\{I_{k}\right\}$.

The basic mechanism for the correlations of RTs in a slowly driven system is illustrated in Fig. 1. Let us assume that the perturbation $\eta(t)=\sigma$ within a time window $[0, T]$, where $T$ is much larger than the mean RT. Then the RT in the left state will be (in most of the cases) smaller than the mean RT and will be (on average) followed by a longer RT in the right state. Vice versa, a long RT in the right state will be followed by a short RT in the left state. Thus, adjacent RTs will be negatively correlated $\left(\rho_{1}<0\right)$ because short RTs are followed by long ones and vice versa. Going beyond adjacent intervals, we see that, for instance, the first and the third RT in a row will be positively correlated $\left(\rho_{2}>0\right)$ because they are both either longer or shorter than the average RT. More generally, any two intervals that are lagged by an even (odd) number will be positively (negatively) correlated, i.e., $\rho_{2 n}>0, \rho_{2 n+1}<0$, for $n=$ $0,1,2, \ldots$ This coherent pattern, however, is found only as long as these two intervals belong to the time window $[0, T]$ for which $\eta(t)=\sigma$. Because of the random switching of the dichotomous forcing $\eta \rightarrow-\sigma$, this coherence is destroyed for higher lags $n$. As a result, the correlations between intervals decrease with increasing lag. This decay of the SCC will strongly depend on the switching rate of the forcing.
The above holds for a slow dichotomous driving. In order to explore the RT correlations for arbitrary values of the switching rate, we chose a discrete approximation of the system that allows for explicit and simple expressions for the SCC. Nontrivial effects at fast driving predicted by this theory were quantitatively confirmed by numerical simulations of the full dynamics equation (1).

Theory.-For sufficiently small noise intensity $D$, the process (1) can be described by the master equation of a discrete-state dynamics as indicated in Fig. 2. The states in the model are determined by two variables: the state of the dichotomous noise $\eta \in\{-\sigma,+\sigma\}$ (i.e., the state of the effective potential) and the number of transitions $N(t) \in$ $\{1,2,3, \ldots\}$ seen until time $t$ including the initial transition [i.e., $N(0)=1$ as indicated in Fig. 2]. Notice that other choices of discretization are possible, for instance, a fourstate model [11]. By keeping track of the number of transitions $N(t)$, our specific mapping will allow us to calculate the statistics of the ordered sequence of RTs (see below). Without loss of generality, we assume the particle to be after the initial transition in the left minimum. Then for odd (even) $N(t)$, the particle is in the left (right) well. Furthermore, for positive (negative) driving $\eta(t)$, the potential is tilted to the right (left). Given the joint probabilities $p_{n, \pm}(t)=\operatorname{Prob}\{N(t)=n, \eta(t)= \pm \sigma\}$ at time $t$ and the transition probabilities per unit time between the states $W(n, \pm \mid n, \mp)=\lambda$ and $W(n, \pm \mid n-1, \pm)=k_{ \pm}$if $n$ is odd, the master equation for the stochastic dynamics reads

$\dot{p}_{n,+}=-\left(k_{+}+\lambda\right) p_{n,+}+k_{-} p_{n-1,+}+\lambda p_{n,-}$,

$\dot{p}_{n,-}=-\left(k_{-}+\lambda\right) p_{n,-}+k_{+} p_{n-1,-}+\lambda p_{n,+} \quad(n$ odd $)$.

Here and in the following, the roles of $k_{+}$and $k_{-}$have to be exchanged if $n$ is even. According to Kramers' theory for the overdamped limit [2], at weak noise intensity $(D \ll$ $\Delta U \pm \sigma)$ the transition rates can be approximated as $k_{ \pm}=$ $\exp [-(\Delta U \mp \sigma) / D] / \sqrt{2} \pi$. The initial probability is $p_{n, \pm}(0)=0$ for $n>1$ (all of the probability is in the first state). The initial values $p_{1, \pm}(0)$ are determined by the steady state probability to be in the upper or lower state right after a transition from right to left. The latter are found to be $p_{1, \pm}(0)=k_{\mp}\left(\lambda+k_{ \pm}\right) /\left[\lambda\left(k_{+}+k_{-}\right)+\right.$ $\left.2 k_{+} k_{-}\right]$. The statistical properties of the interval sequence

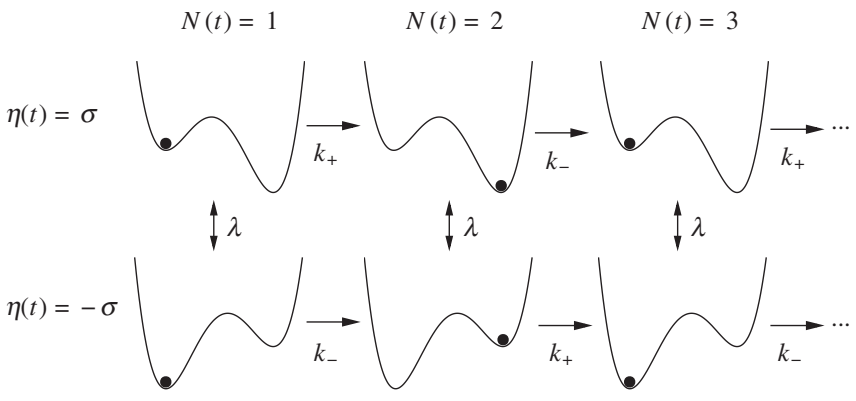

FIG. 2. Cascade of states in the master equation description. 
can be described by the $n$th order interval $T_{n}=\sum_{k=1}^{n} I_{k}$. In order to calculate the statistics of $T_{n}$, we introduce the probability $P_{n}(t)=p_{n,+}(t)+p_{n,-}(t)$ that at time $t$ the particle is in the $n$th state and the probability density $f_{n}(t)=k_{+} p_{n,+}(t)+k_{-} p_{n,-}(t)$ that the $n$th transition occurs at time $t(n$ odd). These quantities allow for a complete description of the process, since the joint probabilities can be expressed as $p_{n, \pm}= \pm\left(f_{n}-k_{\mp} P_{n}\right) /\left(k_{+}+k_{-}\right) \quad(n$ odd). Differentiating this relation and using the master equation (3), we obtain the basic equations for the probabilistic dynamics:

$\dot{f}_{n}=-2(\lambda+k) f_{n}+\left(2 \lambda k+\gamma^{2}\right) P_{n}+\gamma^{2} P_{n-1}$,

$\dot{P}_{n}=-f_{n}+f_{n-1}$,

where $f_{0}=P_{0}=0$. Here we have introduced the geometric mean rate $\gamma=\sqrt{k_{+} k_{-}^{-}}=\exp [-\Delta U / D] / \sqrt{2} \pi$ and the arithmetic mean rate $k=\frac{1}{2}\left(k_{+}+k_{-}\right)=\cosh (\sigma / D) \gamma$. Note that $\gamma$ is independent of the driving and has the meaning of the unperturbed Kramers rate $(\sigma=0)$. The initial conditions for $p_{n, \pm}(0)$ given above imply $f_{1}(0)=$ $\gamma^{2}(\lambda+k) /\left(\lambda k+\gamma^{2}\right), P_{1}(0)=1$, and $f_{n}(0)=P_{n}(0)=0$ for all $n>1$.

The distribution of residence times. - As an example of the standard RT statistics, we discuss now the RT density. The solution of (4) for $n=1$ yields a sum of two exponentials

$$
f_{1}(t)=c_{1} e^{-\alpha_{1} t}+c_{2} e^{-\alpha_{2} t},
$$

with $\quad \alpha_{1 / 2}=k+\lambda \pm \sqrt{k^{2}+\lambda^{2}-\gamma^{2}} \quad$ and $\quad c_{1 / 2}=$ $f_{1}(0) / 2 \pm\left[(k+\lambda) f_{1}(0)-\left(2 k \lambda+\gamma^{2}\right)\right] / 2 \sqrt{k^{2}+\lambda^{2}-\gamma^{2}}$. For a vanishing driving ( $\sigma=0$ leading to $k=\gamma$ ), the RT density is just exponentially decaying $\left(c_{2}=0\right)$ as expected. For a finite $\sigma$ and a large switching rate $\lambda$, the RT density remarkably displays a nonmonotonic behavior which is also confirmed in the simulations of the continuous system [cf. Fig. 3(b)]. For a small switching rate [cf. Fig. 3(a)], the behavior is monotonic. As a matter of fact, in the limit of small $\lambda$ and weak driving $(k / \gamma-1=\varepsilon \ll 1)$, it is hard to extract the value of $\lambda$ from a given density, because corrections to each moment of this density are proportional to $\varepsilon$ in lowest order and $\lambda$ shows up only in higher corrections. This is plausible given that, for instance, at small $\lambda$ we average essentially between two different exponential statistics (with rates $k_{-}$and $k_{+}$) and the exact value of $\lambda$ plays a minor role in the statistics of the single residence time. We will see that the RT correlations, in contrast, are strongly affected by the exact value of $\lambda$, in particular, in the slow driving limit.

The correlation coefficient of the residence times. - The SCC in Eq. (2) can be related to the variances of the $n$th order intervals $T_{n}$ by the formula [10]

$$
\rho_{n}=\left[\left\langle\Delta T_{n+1}^{2}\right\rangle+\left\langle\Delta T_{n-1}^{2}\right\rangle-2\left\langle\Delta T_{n}^{2}\right\rangle\right] /\left(2\left\langle\Delta T_{1}^{2}\right\rangle\right) .
$$

The first and second moments of $T_{n}$ can be calculated from the Laplace transform $\bar{f}_{n}(s)=\int_{0}^{\infty} f_{n}(t) e^{-s t} d t$ as follows:
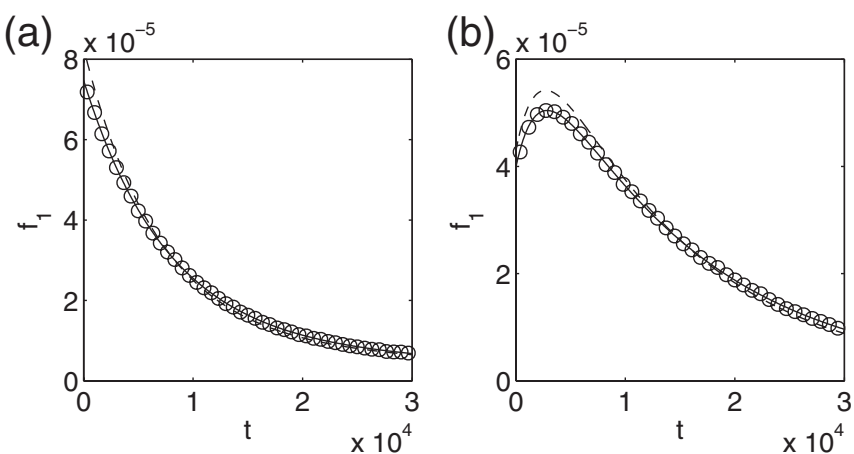

FIG. 3. Residence time densities for $D=\sigma=0.03$ and two different values of $\lambda$ : (a) $\lambda=0.01 \gamma$ and (b) $\lambda=3.4 \gamma$. Simulation results (circles) of Eq. (1) are compared to theory (dashed line) Eq. (5).

$\left\langle T_{n}\right\rangle=-d \bar{f}_{n} /\left.d s\right|_{s=0}$ and $\left\langle T_{n}^{2}\right\rangle=d^{2} \bar{f}_{n} /\left.d s^{2}\right|_{s=0}$, respectively. It is therefore convenient to consider the Laplace transformation of the system (4), which yields the system of linear algebraic equations

$$
\begin{aligned}
{[s+2(\lambda+k)] \bar{f}_{n}-\left(2 \lambda k+\gamma^{2}\right) \bar{P}_{n} } & =f_{n}(0)+\gamma^{2} \bar{P}_{n-1}, \\
\bar{f}_{n}+s \bar{P}_{n} & =P_{n}(0)+\bar{f}_{n-1},
\end{aligned}
$$

where $\bar{f}_{0}=\bar{P}_{0}=0$. Solving for $n=1$, we obtain the moments of the residence times; in particular, the mean RT reads

$$
\left\langle T_{1}\right\rangle=\frac{k+\lambda}{k \lambda+\gamma^{2}} .
$$

For $n>1$, the system (7) can be written in the form of the recurrence relation

$$
\left(\begin{array}{c}
\bar{f}_{n} \\
\bar{P}_{n}
\end{array}\right)=g(s)\left(\begin{array}{cc}
2 k \lambda+\gamma^{2} & \gamma^{2} s \\
2(k+\lambda) & -\gamma^{2}
\end{array}\right)\left(\begin{array}{c}
\bar{f}_{n-1} \\
\bar{P}_{n-1}
\end{array}\right),
$$

with $g(s)=\left[s^{2}+2(k+\lambda) s+2 k \lambda+\gamma^{2}\right]^{-1}$. This relation allows one to find a general expression for the SCC, which holds for all $n \geq 1$. Starting from Eq. (6), it is possible to show by means of mathematical induction that $\rho_{n}$ is given by the alternating geometric sequence

$$
\rho_{n}=C\left[-\frac{\gamma^{2}}{2 k \lambda+\gamma^{2}}\right]^{n},
$$

with

$C=\frac{\left(k^{2}-\gamma^{2}\right)\left(\gamma^{2}-\lambda^{2}\right)}{2 k \lambda\left(k^{2}+\lambda^{2}+\gamma^{2}\right)+3 \gamma^{2}\left(k^{2}+\lambda^{2}\right)+2\left(k^{2} \lambda^{2}-\gamma^{4}\right)}$.

Surprisingly, the correlations vanish exactly at all lags if $\lambda=\gamma$. For $\lambda>\gamma$, the value of the prefactor $C$ in Eq. (11) becomes negative, and, thus, the correlations reappear but reversed in sign. Adjacent RTs are now positively correlated; a RT and its next but one RT will be negatively correlated. The inversion of the correlations is surprising and could be thought of as an artifact due to the approximations involved in our theory. In simulations of the 


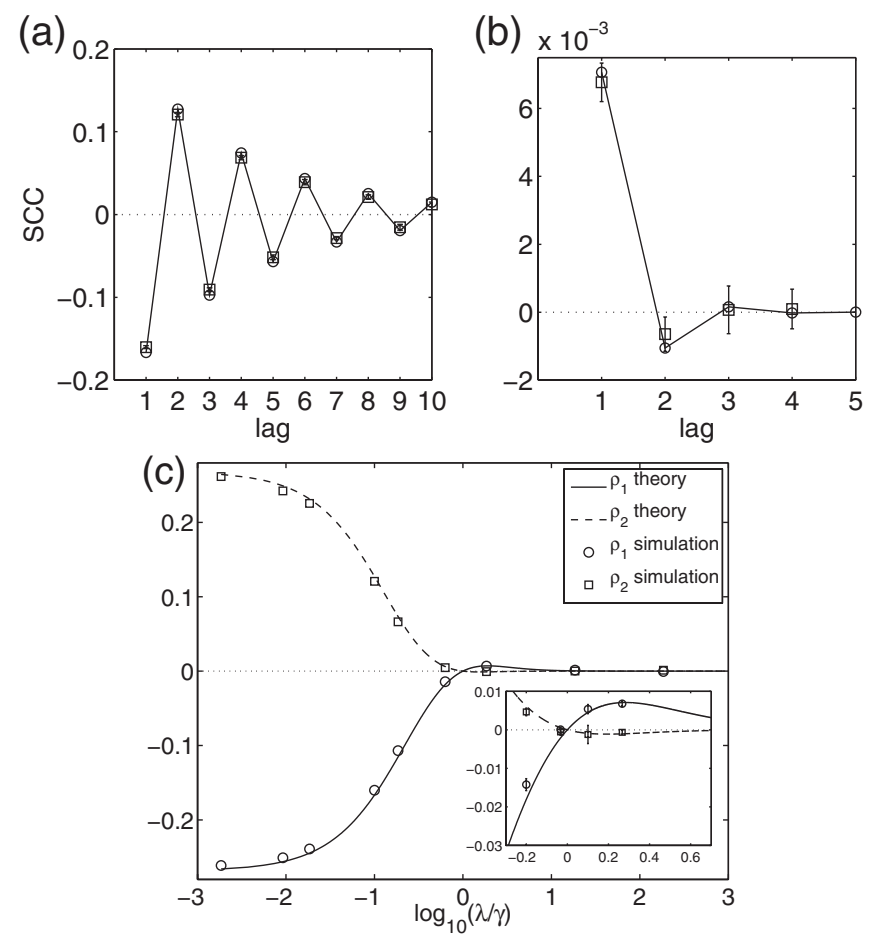

FIG. 4. The SCC as a function of the lag for (a) $\lambda=0.01 \gamma$ and (b) $\lambda=1.8 \gamma$. Note that, going from (a) to (b), the oscillation in the SCC is inverted in sign. The solid line is theory; simulation results are shown by symbols with error bars. (c) shows the first two SCCs vs $\lambda$, obtained from Eq. (10) (solid line) and from simulations of the model equation (1) (symbols). Inset: Magnified view of the data around the zeros of $\rho_{1,2}(\lambda)$.

original continuous model, however, we find exactly the same behavior: at small rate $\lambda$, an oscillating SCC starting with a negative value at lag one, i.e., for adjacent RTs [Fig. 4(a)]. For $\lambda>\gamma$, the correlation is reversed and starts with a positive correlation at lag one [Fig. 4(b)]. Since the correlations for $\lambda>\gamma$ are much weaker than those at small $\lambda$, only the first two lags yield a SCC statistics significantly different from zero. For these two lags, the SCC is shown as a function of $\lambda$ in Fig. 4(c). The simulation results show good agreement with our theory. In particular, we find a value of $\lambda$ very close to $\gamma$ where both SCC values vanish as was predicted by our theory for $\lambda=\gamma$.

It might be of practical interest to know how the SCC is related to the driving parameters; for instance, in situations where the driving is weak, its character (as a dichotomous noise) might be known but not its specific parameters. As a special case, let us consider what happens for a weak and slow dichotomous noise mimicking a long correlated perturbation. Using again the small parameter $\varepsilon$ by $k / \gamma=$ $1+\varepsilon$, with $\varepsilon \ll 1$ (the driving results only in a small asymmetry of the jump process) and $\lambda \ll \gamma$ (the driving is slow compared to the unperturbed jump process), we can write the SCC as follows:

$$
\rho_{n}=2 \varepsilon\left[-\frac{1}{1+2 \lambda\langle T\rangle}\right]^{n}
$$

Given a sequence of residence times, we can easily determine the mean interval $\langle T\rangle$ and the ratio $\rho_{1} / \rho_{2}$. The above relation will then allow us to estimate the switching rate $\lambda$ of the driving process and subsequently (through $\rho_{1}$ ) also the asymmetry parameter $\varepsilon$. We recall that the moments of the RT do not offer similar information about $\lambda$.

Conclusions. - We have calculated the correlations of the residence times of a driven bistable process and have demonstrated that RT correlations may reveal features of the system's dynamics and of the driving that are not present in the statistics of the RT density on which the overwhelming body of work on escape processes has focused so far. In cases where the statistics of the driving are not known, this may help estimate it. We have shown how this could be done for a weak and slow random dichotomous driving.

Serial correlations in the sequence of first-passage times have been analytically studied in Refs. $[9,10]$ for perfect integrate-and-fire (PIF) models of neural firing. Remarkably, one also finds an exponentially decaying correlation in these models for a slowly changing exponentially correlated forcing. However, besides all of the essential differences between the PIF models and our bistable model, these simpler models do not show two important correlation features found here: (i) The correlations do not oscillate with the lag; (ii) the correlations do not vanish and are not inverted at a large switching rate.

We thank Lutz Schimansky-Geier for his support of this study.

[1] P. Hänggi, P. Talkner, and M. Borkovec, Rev. Mod. Phys. 62, 251 (1990).

[2] H. A. Kramers, Physica (Utrecht) 7, 284 (1940).

[3] P. Hänggi and P. Jung, Adv. Chem. Phys. 89, 239 (1995).

[4] V. N. Smelyanskiy, M. I. Dykman, and B. Golding, Phys. Rev. Lett. 82, 3193 (1999); J. Lehmann, P. Reimann, and P. Hänggi, Phys. Rev. Lett. 84, 1639 (2000).

[5] C. R. Doering and J. C. Gadoua, Phys. Rev. Lett. 69, 2318 (1992); P. Reimann, Phys. Rev. Lett. 74, 4576 (1995).

[6] M. H. Choi, R. F. Fox, and P. Jung, Phys. Rev. E 57, 6335 (1998); B. Lindner, J. Stat. Phys. 117, 703 (2004).

[7] B. Lindner, J. Garcia-Ojalvo, A. Neiman, and L. Schimansky-Geier, Phys. Rep. 392, 321 (2004).

[8] M. J. Chacron, A. Longtin, M. St-Hilaire, and L. Maler, Phys. Rev. Lett. 85, 1576 (2000); M. J. Chacron, K. Pakdaman, and A. Longtin, Neural Comput. 15, 253 (2003).

[9] J. W. Middleton, M. J. Chacron, B. Lindner, and A. Longtin, Phys. Rev. E 68, 021920 (2003).

[10] B. Lindner, Phys. Rev. E 69, 022901 (2004).

[11] A. Neiman, L. Schimansky-Geier, F. Moss, B. Shulgin, and J. J. Collins, Phys. Rev. E 60, 284 (1999).

[12] J.F. Lindner and A.R. Bulsara, Phys. Rev. E 74, 020105(R) (2006).

[13] S. Fauve and F. Heslot, Phys. Lett. 97A, 5 (1983).

[14] I. Broussell, Ivan L'Heureux, and Emery Fortin, Phys. Lett. A 225, 85 (1997). 